\title{
Developing an Approach for Analyzing and Verifying System Communication
}

\section{IEEE Aerospace Conference 2009}

\author{
William C. Stratton (JHU/APL) \\ Mikael Lindvall (FC-MD), Chris Ackermann (FC-MD), \\ Deane E. Sibol (JHU/APL), Sally Godfrey (GSFC)
}

Johns Hopkins University/Applied Physics Laboratory Space Department Ground Applications Group (JHU/APL)

Fraunhofer Center for Experimental Software Engineering Maryland (FC-MD)

Goddard Space Flight Center (GSFC)

NASA IV\&V support through a Software Assurance Research Project (SARP) 


\section{Motivation}

- Software systems in the aerospace domain...

- are inherently complex,

- operate under tight resource constraints,

- exist in systems of systems that communicate with each other to fulfill larger tasks

- Reliable systems of systems require reliable communications, but ensuring reliable communications is difficult:

- systems developed independently

- ambiguities in the specification of expected communication behaviors

- issues in communications are often subtle and can go undetected

- Communications problems can lead to waste of space link bandwidth and other precious mission resources 


\section{Organizational Approach}

- $\quad$ NASA IV\&V Software Assurance Research Program (SARP)

- Supports development of software engineering processes and tools

- Encourages collaboration between researchers and practitioners

- $\quad$ FC-MD researchers develop new processes and tools to address communications problems

- JHU/APL practitioners provide communications scenarios and test data for experimentation

- $\quad$ FC-MD and JHU/APL work as one team, using an iterative process...

- Experiment with technology; apply to FC-MD testbed

- Evaluate technology; apply it to APL's ground software systems

- Improve technology based on feedback, results

- Repeat

- Emerging processes and tools extend to NASA projects

- e.g. through the SARP Research Infusion program 


\section{Technical Approach}

- $\quad$ Develop DynSAVE to detect communications problems among systems by analyzing their communication behavior:

- Build on Fraunhofer's proven Software Architecture Visualization and Evaluation (SAVE) tool and process for static analysis of source code

- Enhance for dynamic analysis of run-time communication behavior $=>$ Dynamic SAVE (DynSAVE)

- The DynSAVE approach consists of three steps:

1. Monitor and record low level network traffic

2. Convert low level traffic into meaningful application messages

3. Visualize messages such that issues can be detected 


\section{SAVE Tool and Process}

- $\quad$ SAVE supports static analysis:

- $\quad$ software architect creates models of the planned relationships among abstract software components

- $\quad$ SAVE tool parses source code and lifts the actual relationships among concrete software components

- SAVE tool annotates the architect's models to show deviations from the plan

- $\quad$ software architect uses the SAVE tool to explore the deviations, drilling down through the annotations to the source code

- $\quad$ source code and/or model are updated to eliminate the deviations

- JHU/APL and FC-MD have infused SAVE into the ground software development process:

- $\quad$ used to analyze changes to legacy Common Ground software

- $\quad$ incorporated into new software development for next generation of JHU/APL ground software systems beginning with Radiation Belt Storm Probes (RBSP) 


\section{SAVE \\ Tool and Process}

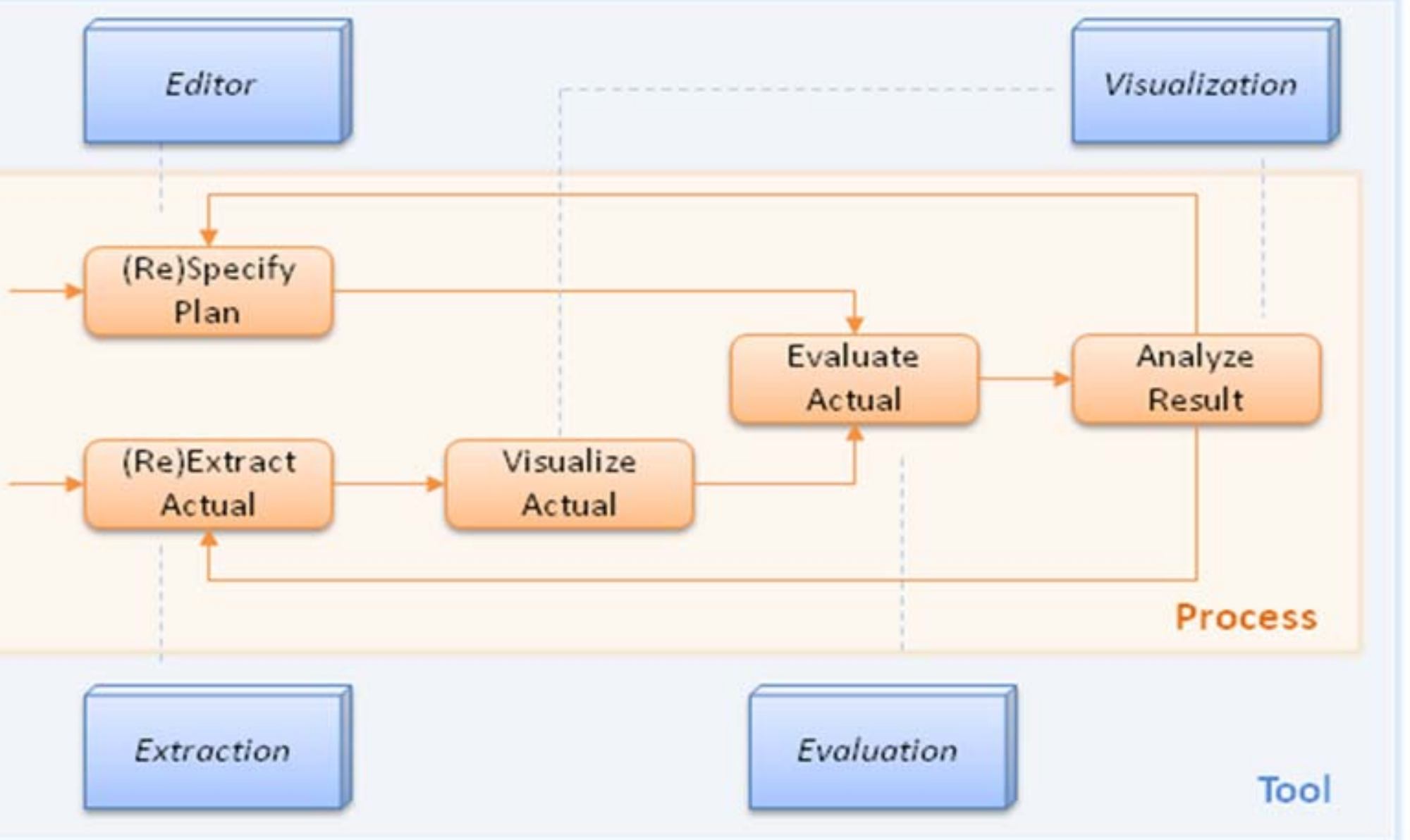


- $\quad$ DynSAVE extends SAVE to support dynamic analysis:

- $\quad$ software architect creates models of the planned message sequences among abstract systems

- actual messages are captured from network traces or low level communications archives

- DynSAVE tool parses captured messages and lifts the actual message sequences among concrete systems

- DynSAVE tool annotates the architect's models to show deviations from the plan

- $\quad$ software architect uses the DynSAVE tool to explore the deviations, drilling down through the annotations to the messages

- $\quad$ systems and/or model are updated to eliminate the deviations

- JHU/APL and FC-MD have applied DynSAVE to mission data systems:

- used to analyze legacy Common Ground software client/server communications (Aerospace 2008)

- currently analyzing CCSDS File Delivery Protocol (CFDP) communications behaviors in RBSP and MErcury Surface, Space ENvironment, GEochemistry, and Ranging (MESSENGER) 
Dynamic SAVE allows for structural and behavioral Architectural analysis of systems of systems

Dynamic Structure

The

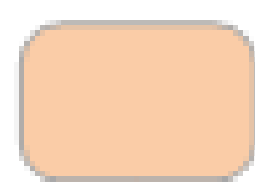

Satellite

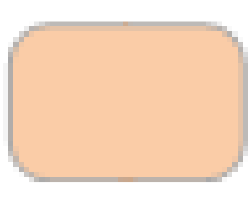

Client B
Client A

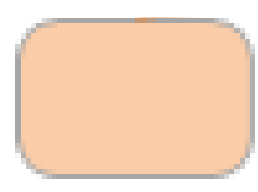
Satellite Seqtignce Diagrams

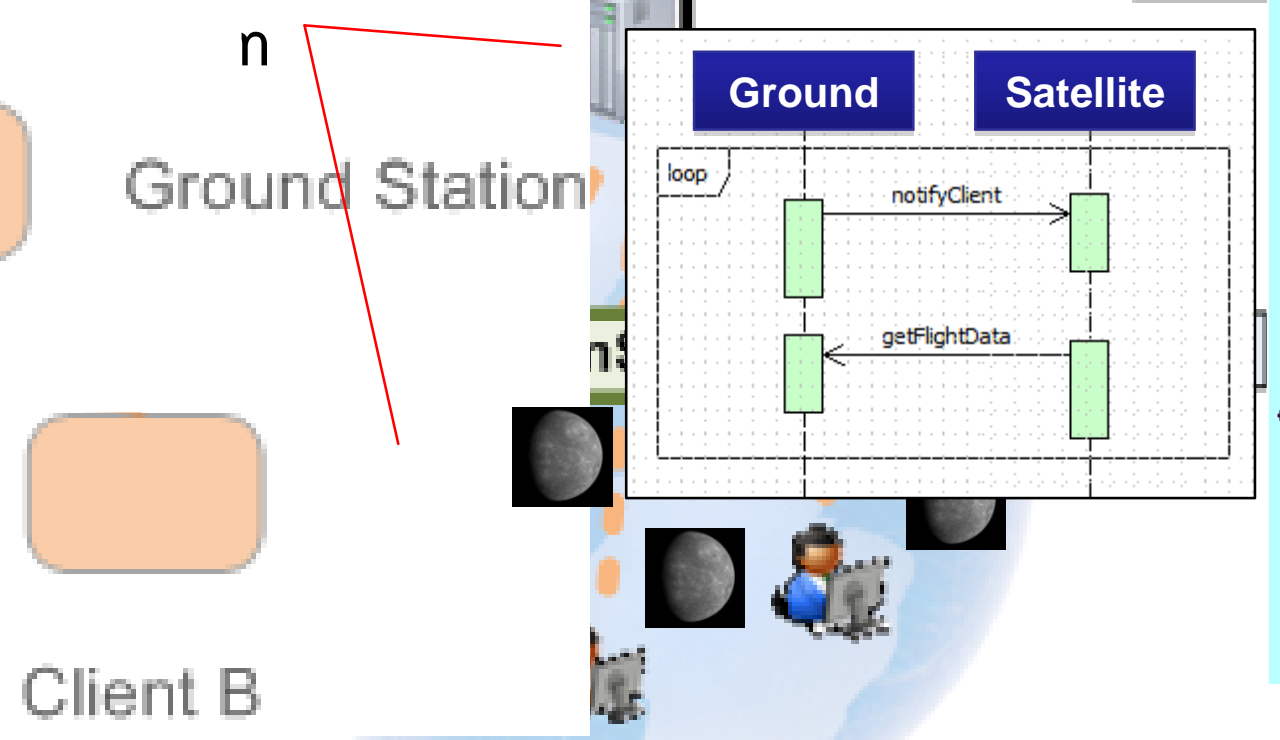

External Users
Actual Behavior

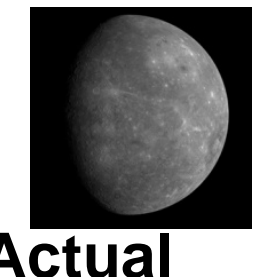

Ground

Satellite 


\title{
Test Data
}

RBSP

Missions

Operation

Center
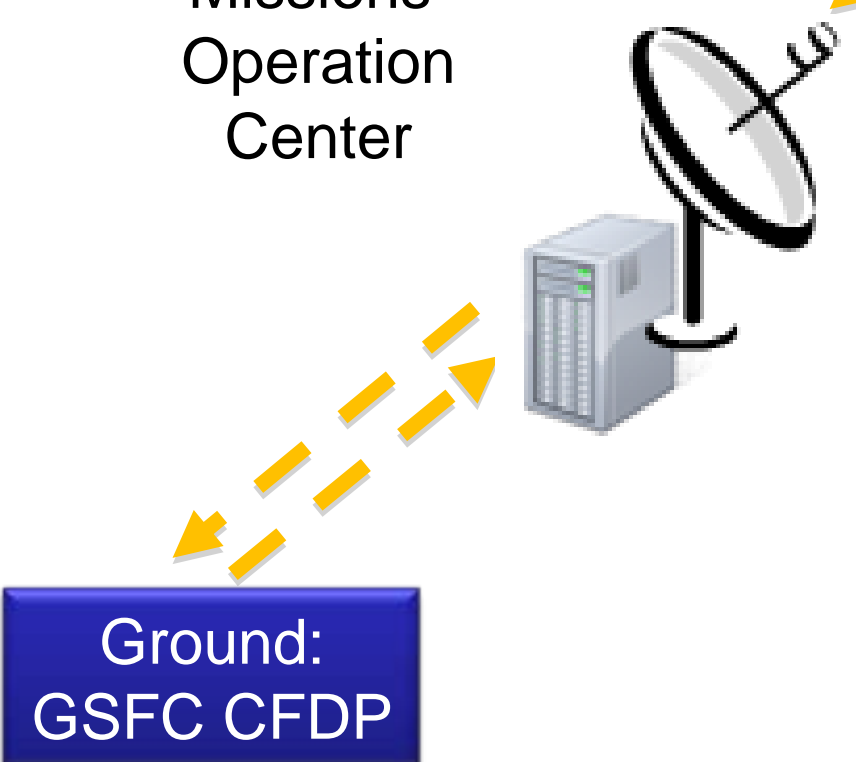

\author{
Does \\ implementation \\ behave as \\ expected?
}




\section{Test Data}

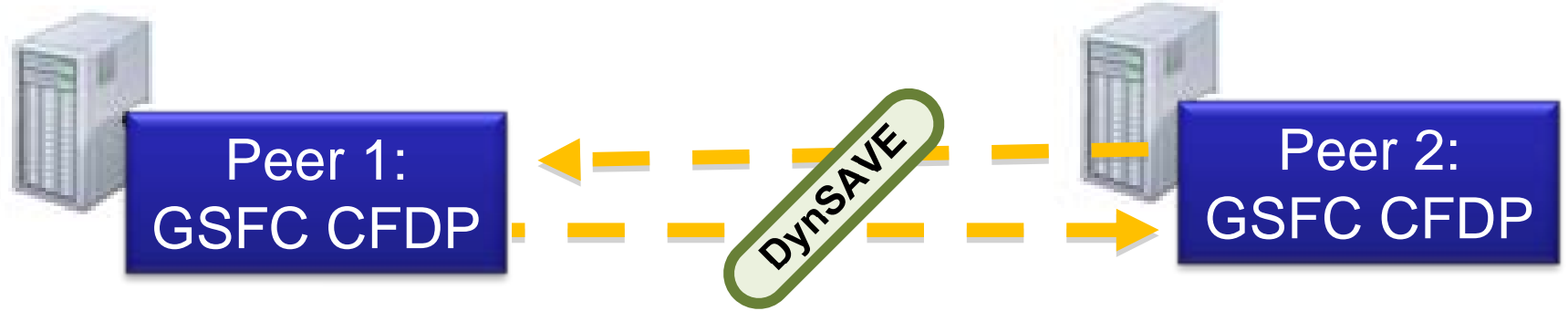

Does implementation behave as expected? 


\section{CFDP - A Mission Data System Protocol}

- CFDP software provides reliable downloads of recorded on-board data

- The implementation is distributed across flight and ground systems

- The protocol runs on top of unreliable CCSDS command and telemetry layer

- At APL, CFDP is mostly automated, but...

- Operators turn off CFDP uplink during critical command load sequences

- Operators freeze and thaw timers so that pending transactions don't time out between contacts

- Improper CFDP operation can lead to

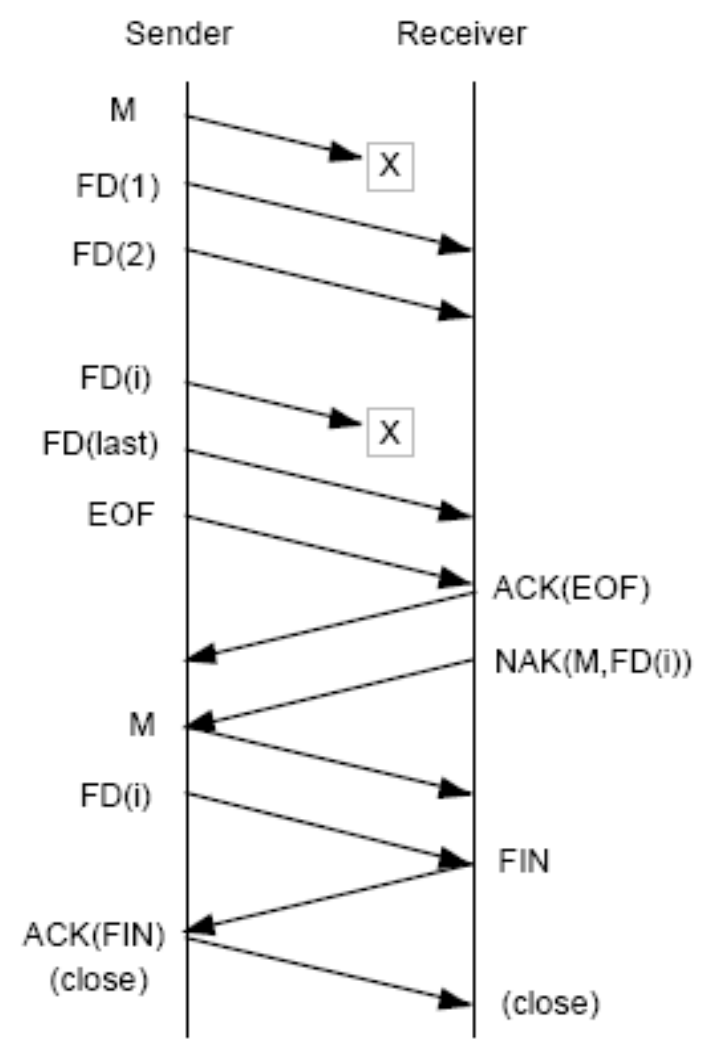
unnecessary retransmissions, wasting precious downlink bandwidth 


\section{DynSAVE monitoring of CFDP}

- DynSAVE monitors macro-level behaviors of the CFDP protocol without affecting flight or ground software

- DynSAVE could detect behaviors that are indicative of improper CFDP operation, for example:

- timers were not frozen and uplink was disabled on the ground for an extended period, causing multiple retransmissions when the uplink was finally enabled again

- DynSAVE could detect behaviors that are indicative of issues in CFDP implementation, for example:

- sender continues to send file data after the transaction has been cancelled

- These types of behaviors can go undetected (file transfers still work) but are important to detect (they can result in data loss!)

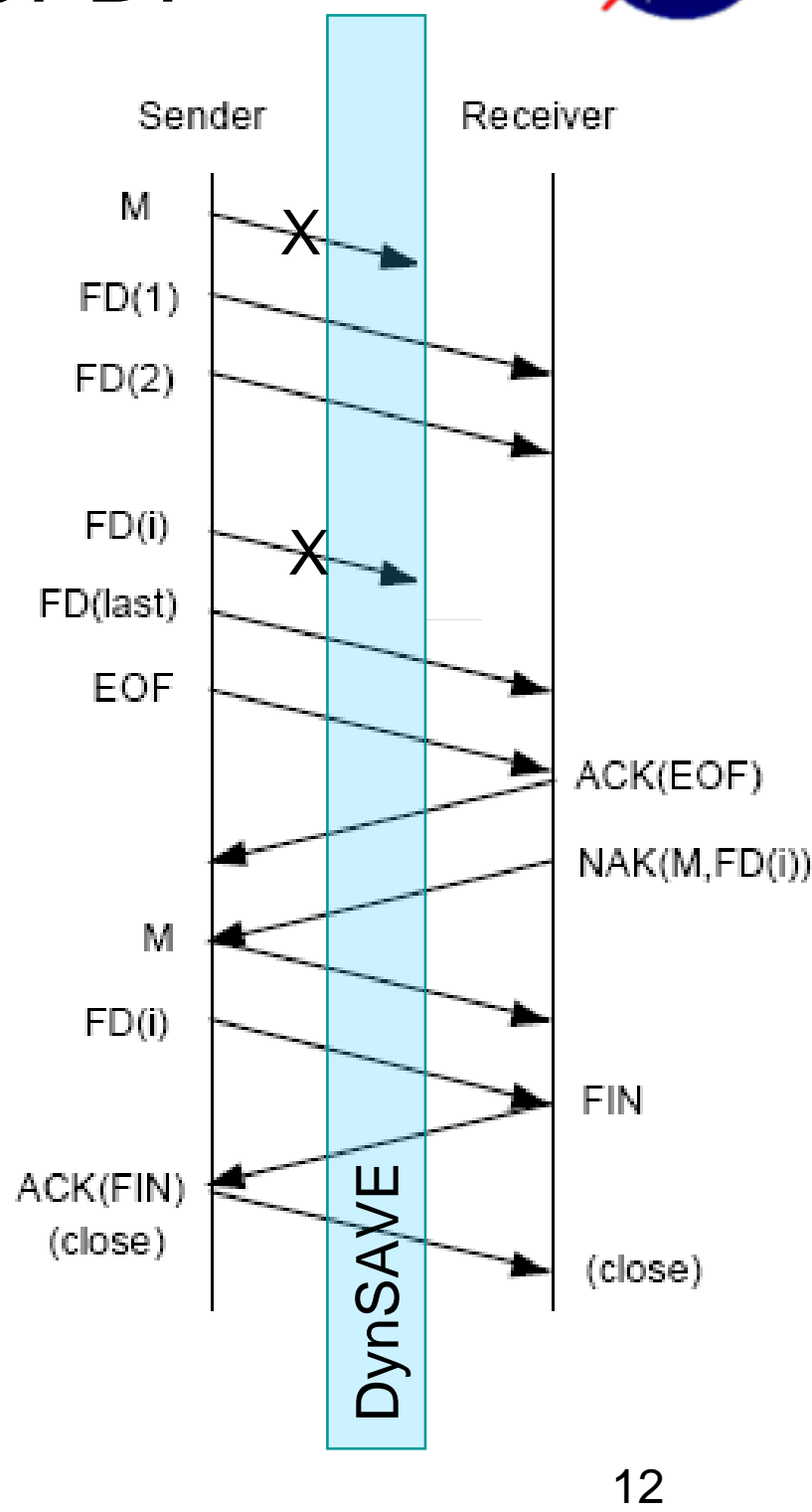




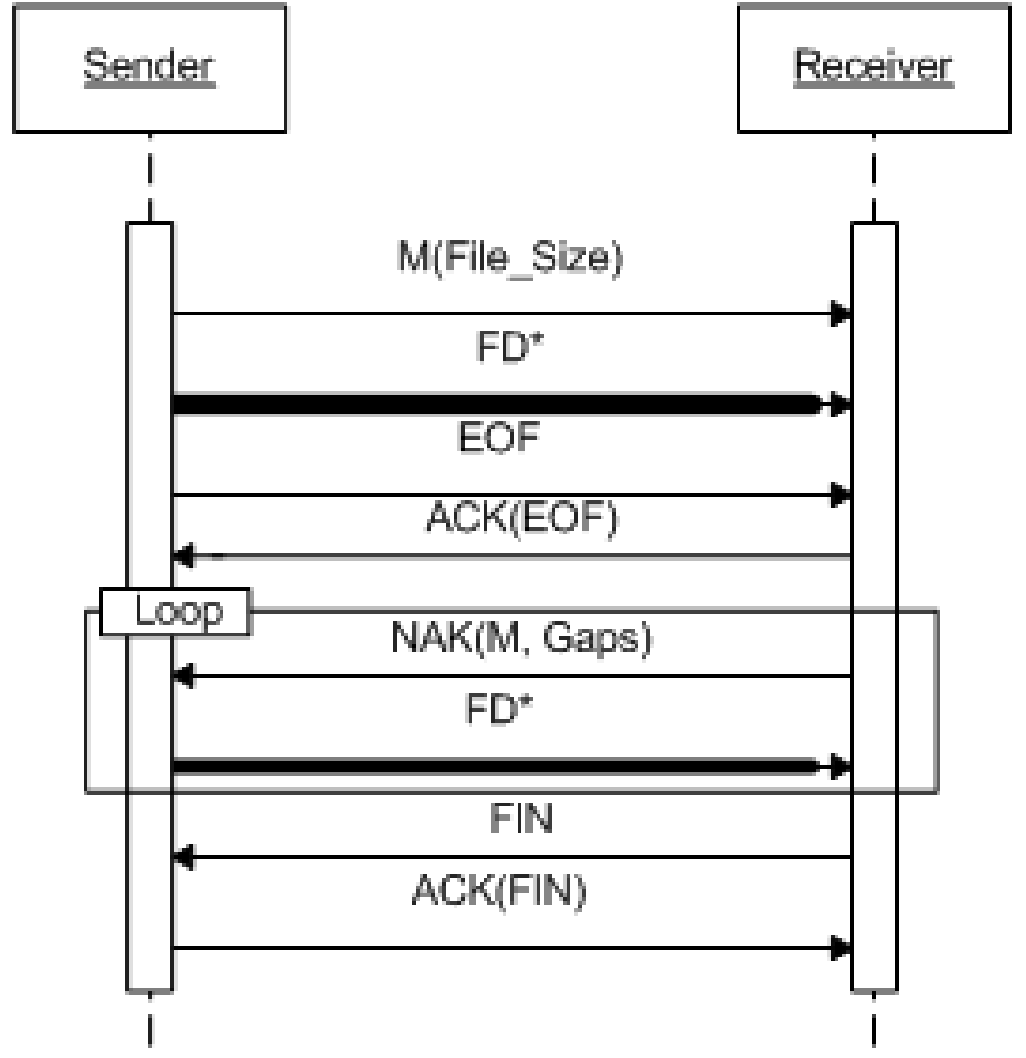

Rules:

1.Check that received FD are not NAKed *

2.Check for duplicate FDs *

3. Check that we have all FDs upon FIN *

4. Check that identical NAKs are not sent back-to-back unless timer went off 


\section{Actual CFDP Sequence}

Metadata: 0-499999

FileData: 0-996

FileData: 997-1993

FileData: $1994-2990$

FileData: 2991-3987

FileData: $3988-4984$

FileData: 4985-5981

FileData: 5982-6978

FileData: 6979-7975

FileData: $7976-8972$

FileData: 8973-9969

FileData: 9970-10966

FileData: 10967-11963

FileData: 11964-12960

FileData: 12961-13957

FileData: 13958-14954

FileData: 14955-15951

Eilenata: 15052-16048 
FileData: 482548-483544

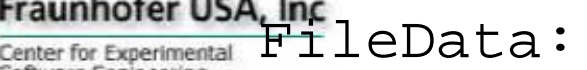

FileData:

FileData:

FileData:

FileData:

FileData:

FileData:

FileData:

FileData:

FileData:

FileData:

FileData:

FileData: 498500-499496

FileData: 499497-499999

EOF: Condition Code $=$ No Error ACK(EOF): Condition Code $=$ No Error

NAK: $19940-20937 ; 27916-28913 ; 36889-37886 ; 56829-$ $59820 ; 72781-73778 ; 76769-77766 ; 82751-85742 ; 101694$ $102691 ; 111664-112661 ; 115652-116649 ; 121634-$ $122631 ; 130607-131604 ; 139580-140577 ; 146559-$ $147556 ; 153538-154535 ; 155532-156529 ; 170487-$

$171484 ; 197406-198403 ; 203388-204385 ; 220337-498500$ 


\section{Mapping CFDP data}

- The sniffed CFDP data is low level (packets)

- Concepts are often encoded

- Few message names in clear text

- Many are not: e.g. Cancel

- If third bit in EOF control message then Cancel

- Parameters are always encoded

- E.g. bit 4-16: Time stamp

- Communications are often interleaved

- E.g. Files sent and received concurrently

- Our parser maps low level data to high level messages and values, identifies \& separates interleaved communications 
Actual CFDP Sequence captured in test lab

Sample Rule:

Never re-request a package that already was received

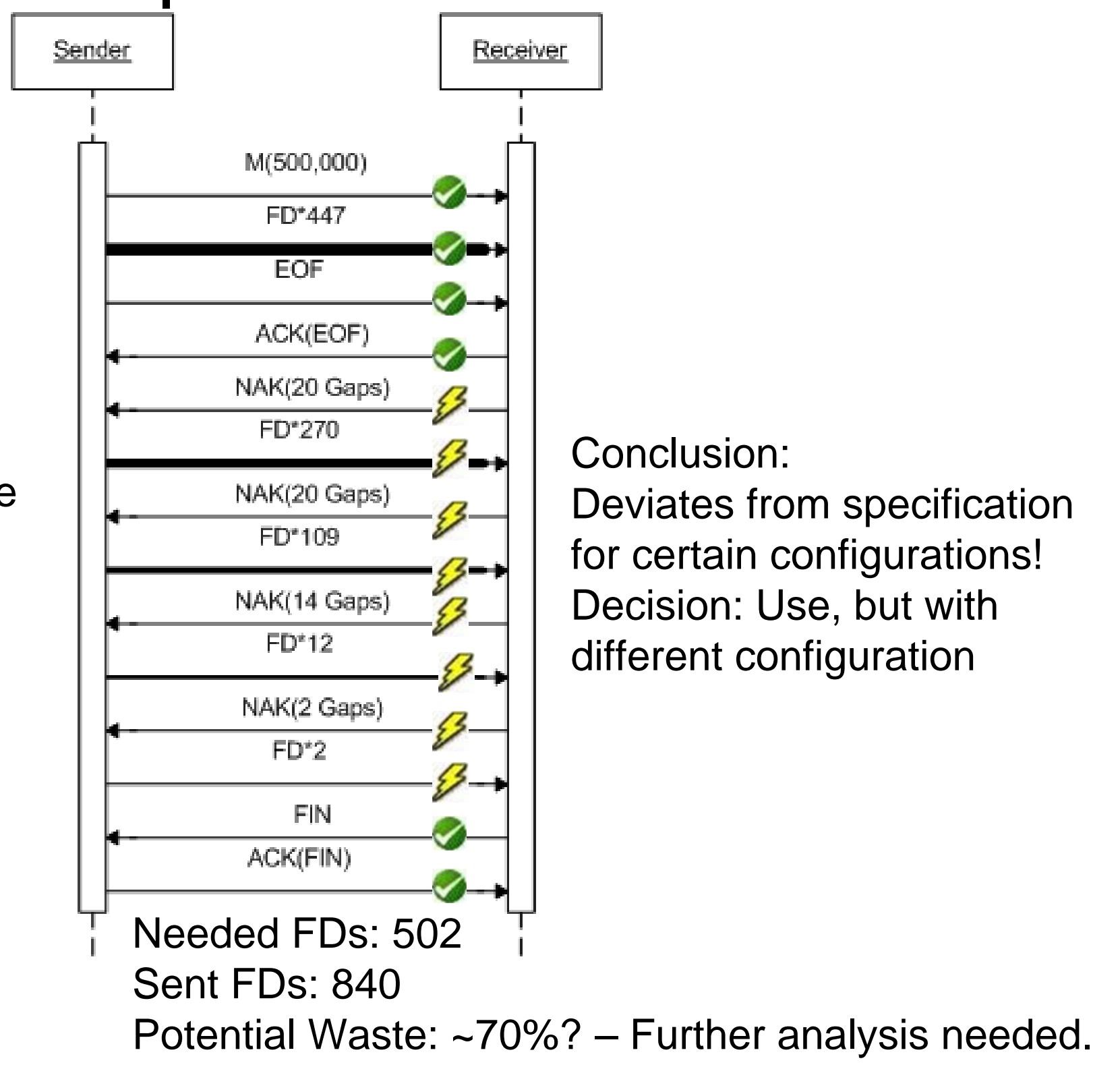


F曲 Fraunhofer USA, Inc

Center for Experimental
softrore Engneering
swat

Zoom in on CFDP sequence

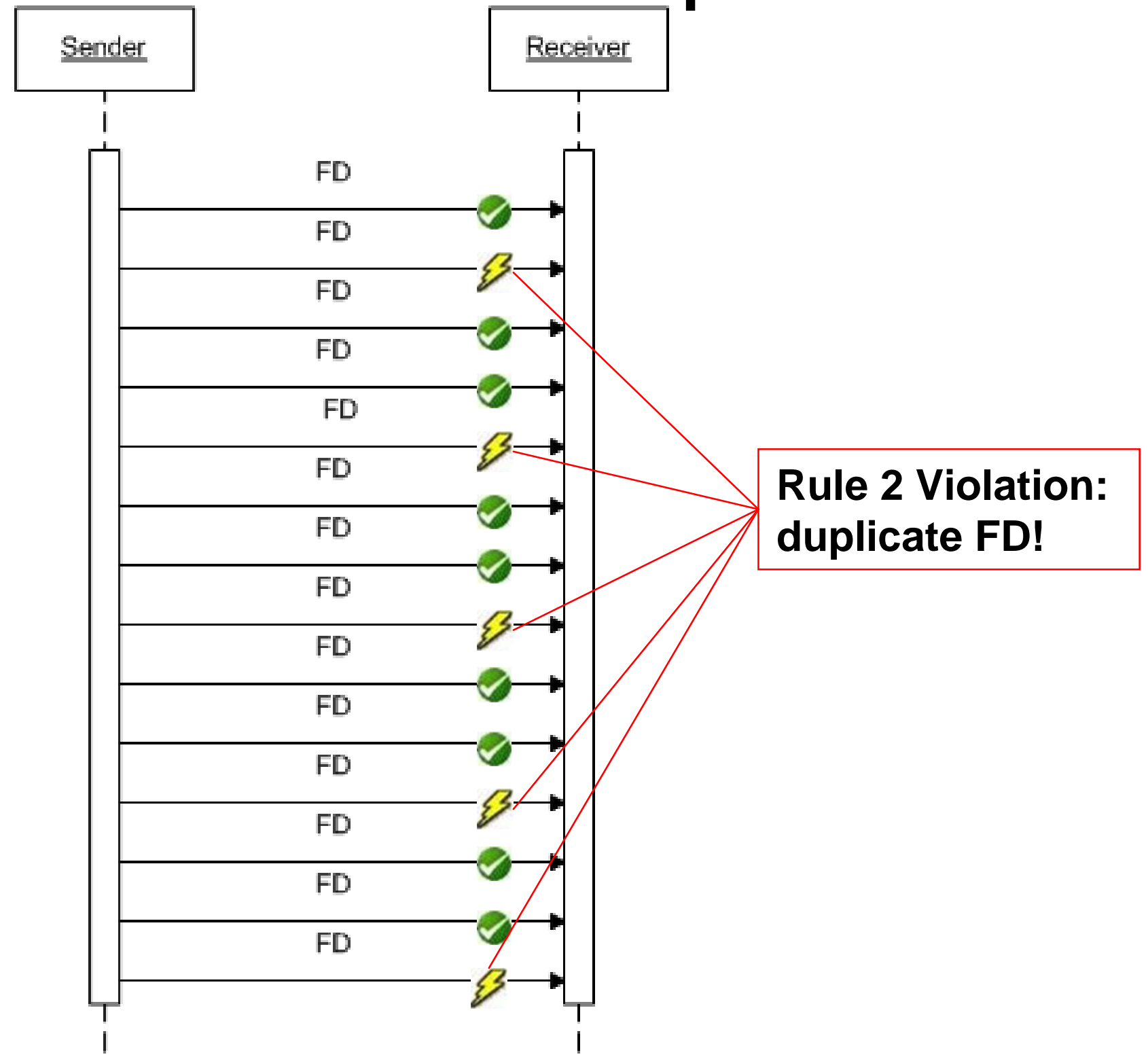




\section{Live Data}

MESSENGER

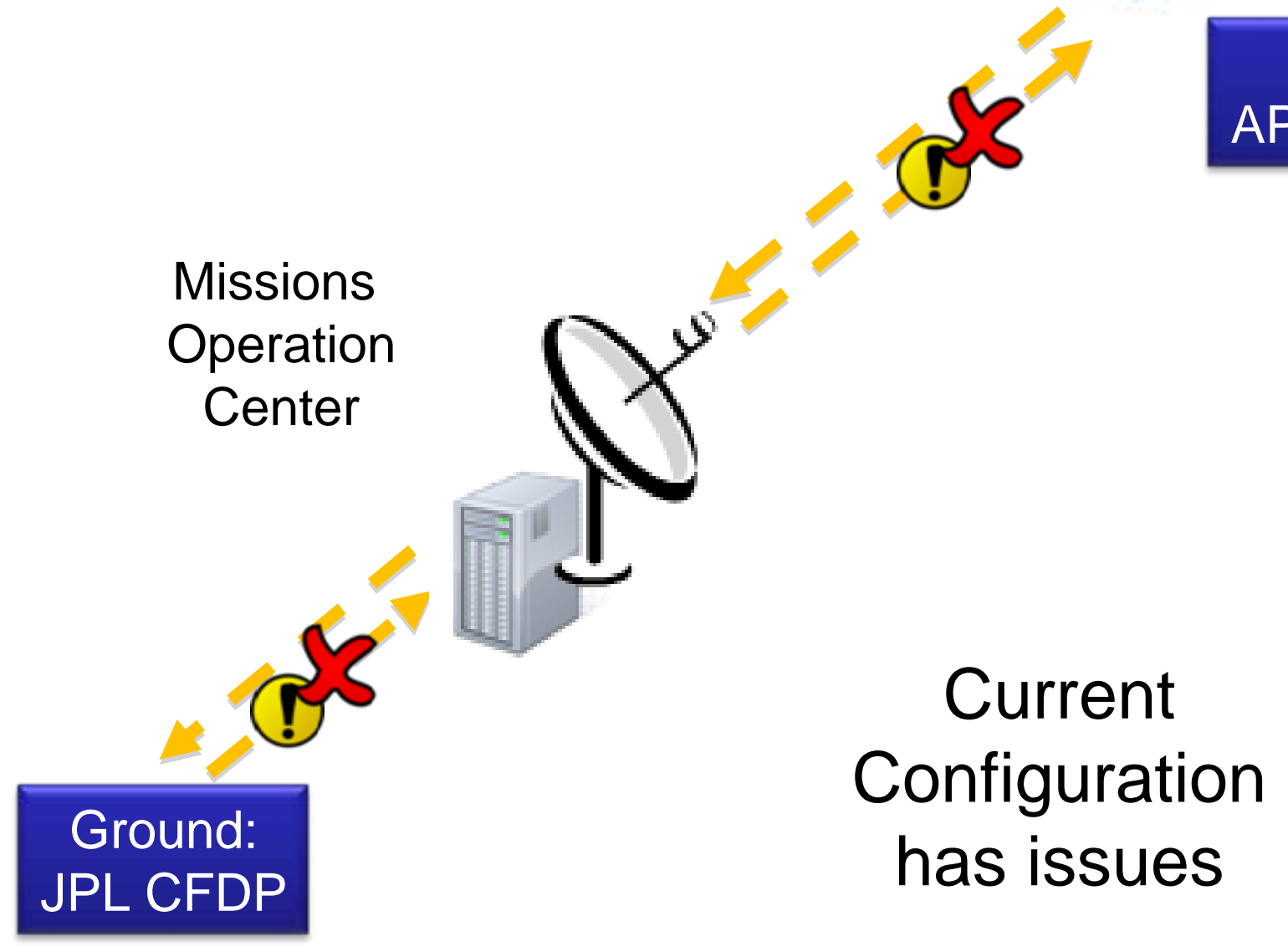

Missions

Ceration

Center

Configuration

has issues 


\title{
Strategy: Shadow Mode
}

\author{
MESSENGER
}

\author{
Flight: \\ APL CFDP
}

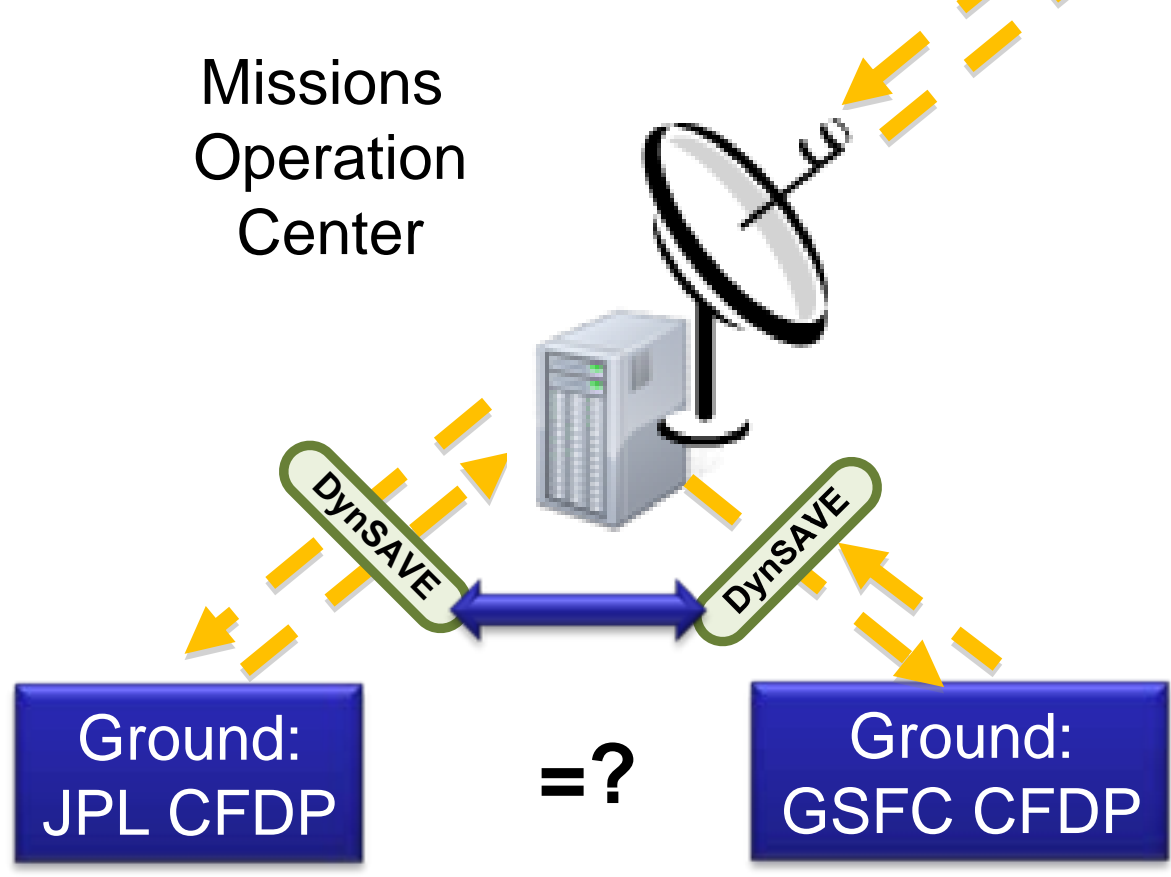

Test to be conducted in May 2009 


\section{Life Cycle Support}

Initial use of Dyn SAVE

\begin{tabular}{|c|c|c|}
\hline $\begin{array}{c}\text { System } \\
\text { Architecture }\end{array}$ & $\begin{array}{l}\text { Sub-System } \\
\text { Development }\end{array}$ & $\begin{array}{l}\text { System } \\
\text { Integration and Test }\end{array}$ \\
\hline $\begin{array}{l}\text { Use DynSAVE to } \\
\text { Specify and Test } \\
\text { Communication } \\
\text { Add to ICD }\end{array}$ & $\begin{array}{l}\text { Use DynSAVE to } \\
\text { Develop and Test } \\
\text { based on ICD }\end{array}$ & $\begin{array}{l}\text { Use DynSAVE to } \\
\text { test based } \\
\text { on ICD }\end{array}$ \\
\hline
\end{tabular}




\section{Summary}

- Analyze, Visualize, and Evaluate

- structure and behavior using static and dynamic info of

- individual systems as well as systems of systems

- Drive R\&D by needs from JHU/APL NASA missions

- Use open testbed for experimentation

- Evaluate together with APL in their context

- Transfer technology when mature

- Future:

- Add time information and constraints (current activity)

- Add planned sequence diagrams to ICD

- Use for analysis of Delay Tolerant Network Management 